\title{
Medical art report: an instrumental song of hope to end the COVID-19 pandemic
}

(News report: Art in Medicine)

Type of article: Medical art

\author{
Abstract \\ NA: This is a short news report and does not have an abstract. \\ Keywords: COVID-19, Music, COVID song, COVID art, Instrumental song, Pandemic
}

"End of the Pandemic" is an instrumental song or piece in the Pop-Electronic genre that was released in 2021, when the entire world was facing the worst waves of the COVID-19 Pandemic. This song was created based on a scientific study that employed qualitative research methods (interviewing physicians and patients about their feelings and thoughts about the pandemic) and a review of the literature. The piece's main researcher and producer used the research results to translate people's feelings about the COVID-19 Pandemic into music. "End of the Pandemic" is a song of hope, resilience, and tolerance, composed and produced by Dr. Mehrdad Jalalian and Afshin Rezaei; and a meaningful background picture designed by Richard Borge was used in the YouTube version of the music (Figure 1). The music is available on YouTube at https://youtu.be/wrMC k66-QQ

There are three parts to the song: 1) The first part represents normal life prior to the covid pandemic, 2) the second part describes the covid pandemic and its waves, as well as the patients' and physicians' reactions to the pandemic and the disease itself, including stress, anxiety, fears, sadness, and grief; and finally, the song's third part begins with a miraculous event that brought the pandemic to an end. The miracle is brought to the world by scientists (who generated the vaccine) and by people and their responsible behaviors such as social distancing, mask wearing, vaccination, and other measures.

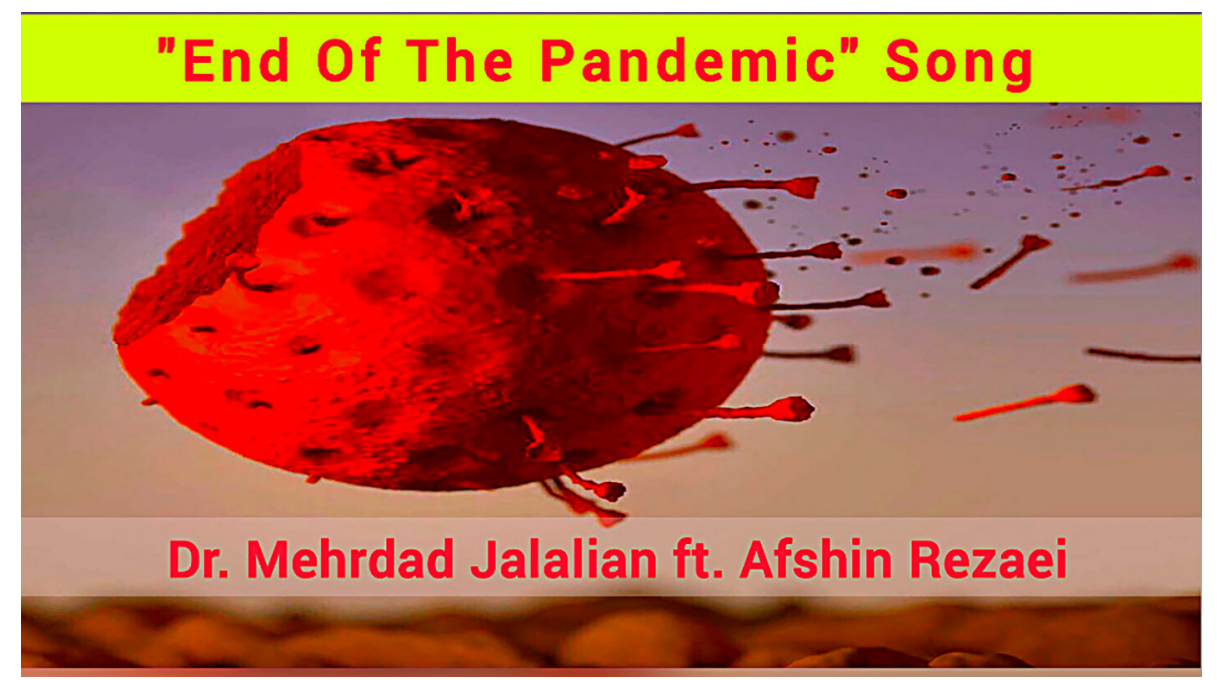

Figure 1. Background picture of "End of the Pandemic" instrumental song, designed by Richard Borge 